\title{
Occupation Humaine De La Réserve De Faune Et De Flore Du Haut Bandama : Diagnostic Et Perspectives Pour Une Gestion Durable
}

\author{
Dr Dien Kouayé Olivier \\ Centre de Recherche en Ecologie (CRE), \\ Université Nangui Abrogoua, Côte d'Ivoire
}

Doi:10.19044/esj.2018.v14n26p93 URL:http://dx.doi.org/10.19044/esj.2018.v14n26p93

\begin{abstract}
The Upper Bandama fauna and flora reserve, which is part of Côte d'Ivoire's biodiversity conservation strategy, is threatened by the pressure of human activities. The purpose of this article is to make a socio-economic assessment of the exploitation of the natural resources of this protected area. Specifically, it is question of knowing the forms of activities which are developed there, the actors, the strategies of infiltration, and to determine the factors related to this occupation. Based on a data collection work that combines documentary research, semi-structured interviews and direct observation, artisanal gold mining, farming, poaching, fishing and collection of non-timber forest products have been identified as the major forms of degradation of this reserve. These activities involve different profiles of actors who have accessed the reserve under various conditions. They can be explained by the economic dynamism of the peripheral zone, the recomposition of this zone in the face of demographic pressure and land issues, the non-integration of the legal status of the reserve and the invisibility of the key actors. The study ends with perspectives for sustainable management of this reserve.
\end{abstract}

Keywords: Natural Resources, Protected Areas, Environmental Crime, Upper Bandama Reserve

\section{Résumé}

La réserve de faune et de Flore du Haut Bandama, outil qui s'intègre dans la stratégie de conservation de la biodiversité de la Côte d'Ivoire, se trouve être menacée par la pression des activités humaines. L'objectif de cet article est de faire un état des lieux socio-économique de l'exploitation des ressources naturelles de cette aire protégée. De façon spécifique, il s'agit de connaître les formes d'activités qui y sont développées, les acteurs, les 
stratégies d'infiltration, et de déterminer les facteurs liés à cette occupation. A partir d'un travail de recueil de données qui associe recherche documentaire, entretiens semi-directifs et observation directe, l'orpaillage, l'élevage, l'agriculture, le braconnage, la pêche et la collecte de produits forestiers non ligneux ont été identifiés comme les grandes formes de dégradation de cette réserve. Ces activités impliquent différents profils d'acteurs qui ont accédé à la réserve dans diverses conditions. Elles s'expliquent par le dynamisme économique de la zone périphérique, la recomposition de cette zone face à la pression démographique et aux enjeux fonciers, la non intégration du statut juridique de la réserve et l'invisibilité des acteurs clés. L'étude se termine par des perspectives pour la gestion durable de cette réserve.

Mots clés : Ressources naturelles, Aires protégées, Criminalité
environnementale, Réserve du Haut Bandama

\section{INTRODUCTION}

La création d'aires protégées est aujourd'hui reconnue comme un outil fondamental pour parvenir à la protection de l'environnement en général et des écosystèmes en particulier. En Côte d'Ivoire, les efforts consentis dans ce sens ont abouti à la mise en place d'un réseau de huit (8) parcs nationaux et six (6) réserves naturelles. Bien que ne couvrant que 6,5\% du territoire national (soit 2,1 millions d'hectares), l'essentiel de la diversité biologique de la Côte d'Ivoire est concentré dans ces aires protégées qui hébergent près de $90 \%$ des espèces de mammifères et d'oiseaux connues dans la région Ouest africaine et qui constituent, avec les forêts classées, la majeure partie du couvert végétal naturel subsistant sur le territoire ivoirien (BERD-E\&D Consult, 2014).

Ces parcs nationaux et réserves naturelles sont placées sous le contrôle de l'Etat et leurs limites ne peuvent être changées, ni aucune partie aliénée, sauf par l'autorité législative compétente (article $1^{\mathrm{er}}$ de la Loi $\mathrm{n}^{\circ} 2002-102 \mathrm{du} 11$ février 2002 relative à la création, à la gestion et au financement des parcs nationaux et Réserves naturelles). Mais, depuis quelques années, ces espaces censés être protégés sont affectés par de multiples impacts résultant de la fréquence des feux de brousse et de la pénétration et de l'installation des personnes humaines pour des activités agricoles, pastorales ou extractives de matières premières (DSRP, 2009).

Cette situation défavorable a amené le Gouvernement ivoirien à engager, à partir de 1995 avec les partenaires au développement, la réforme de la gestion des parcs nationaux et réserves naturelles. Ce processus a été couronné par l'élaboration du Programme-Cadre de Gestion des Aires Protégées (PCGAP). La mise en œuvre de ce programme s'est traduite, entre autres, par le renforcement du cadre juridique du secteur des parcs nationaux et réserves 
naturelles à travers l'adoption de la Loi n ${ }^{\circ} 2002-102$ sus citée. En application de cette Loi, il a été créé, par Décret n 2002-359 du 24 juillet 2002, l'Office Ivoirien des Parcs et Réserves (OIPR) pour la gestion durable de ce secteur (OIPR, 2016).

Mais la situation des aires protégées de Côte d'Ivoire, déjà préoccupante, s'est, selon différentes études, encore aggravée durant la crise sociopolitique qu'a connue la Côte d'Ivoire où la grande majorité des parcs et réserves naturelles est restée sans aucune mesure de gestion appropriée, suite au retrait de l'administration régulière des zones Centre, Nord et Ouest du pays. Par conséquent, ces espaces ont subi un fort impact destructif avec une poursuite accélérée des défrichements, du braconnage et d'une exploitation non autorisée et non contrôlable.

C'est notamment le cas de la réserve de faune et de flore du Haut Bandama qui, à l'image de plusieurs aires protégées, connaît un recul de près de 30.000 ha de son couvert végétal (Agoualé, 2014) sous l'effet d'une forte agression de la part des populations. Cette réserve, bien qu'intégrée au réseau national des aires protégées, n'avait fait l'objet, depuis sa création, d'aucune véritable prise en charge par une structure de l'administration forestière afin d'en assurer la gestion. Ce n'est qu'en février 2014, après des missions exploratoires qui en ont évalué les potentiels, que l'OIPR a nommé un chef de secteur pour amorcer un début de sa gestion effective (Akindes et al. 2016).

La présente étude, qui se veut une contribution aux efforts de conservation de la biodiversité de cette réserve, a pour objectif de faire un état des lieux socio-économique de l'exploitation des ressources naturelles de ladite aire protégée. Ensuite, prenant en compte les leviers qui agissent sur cet espace, cette étude se termine par des propositions de stratégies de conservation durable de cette réserve. L'essentiel des résultats de ce travail s'insère dans le cadre des activités du projet Droits de Propriété et Développement du Diamant Artisanal (DPDDA II) en Côte d'Ivoire. Ce projet cofinancé par l'USAID et l'Union Européenne est mise en œuvre par la société TETRA TECH.

\section{METHODOLOGIE}

La stratégie arrêtée pour cette étude a consisté à réaliser la collecte d'informations à partir des localités dont le territoire est contigu à la réserve de faune et de flore du Haut Bandama. Située dans le Centre Nord de la Côte d'Ivoire, cette réserve est à cheval sur trois régions (03) régions : Hambol, Poro et Béré ; trois (03) départements : Katiola, Dikodougou et Mankono ; et six (06) Sous-préfectures : Fronan, Niakara, Tortiya, Boron, Marandallah et Bouandougou. Neuf (09) villages issus de ces départements ont fait l'objet d'enquêtes dans le cadre de cette étude. Il s'agit de Namplékaha et Darakokaha dans la sous-préfecture de Fronan, Latokaha et Kafiné dans la sous-préfecture de Niakaramandougou, Ténindiéri, Zanakaha, Sankadjokaha dans la sous- 
préfecture de Tortiya, Tienigbe bobo dans la sous-préfecture de Marandallah et Nakara dans la sous-préfecture de Bouandougou.

Pour atteindre les objectifs de cette étude, nous avons retenu la méthode d'échantillonnage raisonné. Ainsi, les acteurs des secteurs de l'administration et de la conservation de la biodiversité (OIPR), du fait de leur qualité de témoins privilégiés pouvant fournir des données d'opinion et de fait en rapport avec le sujet, ont été interrogés relativement à la dégradation de la réserve et aux perspectives de sa conservation. Outre ces personnes, différentes catégories sociales utilisatrices de la réserve (groupes cibles) ont été concernées par l'enquête.

La population enquêtée se répartit comme suit: 1 Secrétaire Général de Préfecture, 3 Sous-préfets, 2 agents communaux, 5 responsables et agents de l'OIPR, 1 chef de canton, 9 chefs de villages, 13 notables, 7 responsables de communautés allogènes, 4 responsables de communautés allochtones, 2 responsables d'organisations féminines, 5 responsables d'organisations de jeunesse, 13 propriétaires terriens et 132 paysans témoins. Au total, nous avons consulté un échantillon de 197 personnes.

La démarche de cette étude repose sur la méthode systémique et la méthode phénoménologique. La première nous permet d'envisager l'occupation humaine de la réserve de faune et de flore du Haut Bandama comme le produit de plusieurs facteurs en interaction. Elle nous permet d'étudier l'ensemble de ces facteurs ainsi que leurs interrelations dans une perspective globale. La deuxième nous paraît pertinente pour cerner le vécu et les pratiques des acteurs qui occupent la réserve ainsi que les perceptions qu'ils ont de cette aire protégée.

Les trois (03) principales techniques de recueil des données auxquelles nous avons eu recours sont l'analyse documentaire, les entretiens semidirectifs et l'observation.

La recherche documentaire a été faite principalement à la direction de zone de l'OIPR à Yamoussoukro et, au niveau local, dans les services du secteur de l'OIPR de Katiola et des Sous-préfectures riveraines (Marandalla, Tiénigboué, Bouandougou et Fronan). Mais, cette recherche a été contrariée par la pauvreté des informations compilées sur la zone d'étude. Les documents que nous avons pu consulter sont constitués de trois (03) monographies départementales (Katiola, Dikodougou et Mankono), d'un mémoire de master, d'un article et de cartes de la réserve. Ces documents nous ont permis d'orienter certaines informations à recueillir sur le terrain et de les analyser. Ils ont été complétés par des travaux de recherche traitant de certains aspects pris en compte dans notre étude

Les entretiens semi-directifs ont permis à nos interlocuteurs, en l'occurrence les autorités administratives, les gestionnaires de la réserve et les populations locales utilisatrices des ressources naturelles de la réserve, de 
s'exprimer de façon assez libre. Les principales informations recherchées ont porté sur l'historique de la gestion de la réserve, les activités économiques dans et autour de la réserve, les modes d'accès à la réserve, les acteurs, la perception de la réserve, les modes de gestion locale de la terre et des ressources naturelles, la gestion des conflits liés aux ressources naturelles et les facteurs influençant les pressions sur la réserve. Outre ces entretiens formels, nous avons eu recours à des entretiens informels qui se distinguent par un style de conversation non planifié et sans support de prise de notes (Kaboré, 2010).

A travers l'observation directe des activités qui se déroulent dans la réserve, nous avons pu apprécier leur spatialisation et leur portée, ainsi que les acteurs concernés. Cette technique a aussi permis de voir les contradictions qui existent parfois entre les écrits, les dires et des faits sur le terrain.

$\mathrm{Au}$ regard des données obtenues, nous avons privilégié l'emploi de la méthode d'analyse qualitative. Il s'est agi de cerner la nature de l'occupation humaine de la réserve à travers les discours des enquêtés.

\section{RESULTATS}

\section{Les formes d'activités anthropiques dans la réserve de faune et de flore du Haut Bandama}

L'occupation humaine de la réserve de faune et de flore du Haut Bandama couvre plusieurs formes d'activités dont l'orpaillage, l'élevage, l'agriculture, le braconnage, la pêche et la collecte de produits forestiers non ligneux.

\section{L'orpaillage}

L'analyse du discours des agents de l'OIPR en charge de la gestion de la réserve permet d'avoir une idée de la présence des orpailleurs clandestins dans cette aire protégée. Ces derniers ont été en grand nombre à l'amorce de la gestion de cette aire protégée en 2014. Composés majoritairement des étrangers, ils ont eu à leur actif des campements entiers à l'exemple de Seydoukaha et Woyoba.

"Nous avons trouvé une réserve presque totalement conquise par les orpailleurs clandestins. A l'occasion des patrouilles, nous avons interpellé plusieurs d'entre eux et nous avons détruit leur matériel. Mais c'était sans compter avec la détermination de ces gens, qui reviennent exploiter ces sites ou qui les abandonnent au profit d'autres plus cachés. Ce qui est difficile, c'est que ce sont des pratiques qui datent de la crise et dont les auteurs ont une certaine assise au plan local. On ne peut donc pas les maîtriser pour le moment $\gg$.

D'anciens agriculteurs locaux, à l'image des populations du village de Sirikikaha, se sont également reconvertis dans cette activité, qui était réputée procurer des revenus considérables dans l'immédiat. Ces orpailleurs ont creusé plusieurs galeries et puits qui sont visibles sur de grandes surfaces de 
la réserve (figures 1 et 2), en particulier la rive ouest du fleuve Bandama qui traverse la réserve du Nord au Sud. Cette partie se trouve si profondément transformée que la reconstitution de la végétation détruite semble difficile à ce niveau (figure 2). Comme mode opératoire, les orpailleurs extraient l'or par des procédés améliorés avec l'utilisation des engins et des équipements (motopompes, tricycles, barils, groupes électrogènes, broyeurs, etc.). Ils se servent aussi de l'eau du fleuve Bandama pour laver la boue remontée de son lit ou la terre provenant des puits d'extraction. L'afflux d'hommes et de femmes qui se crée autour des sites d'orpaillage, le caractère itinérant des activités, et la nature des techniques et des équipements utilisés font dire aux agents de l'OIPR enquêtés que l'orpaillage constitue la menace la plus importante pour la réserve.
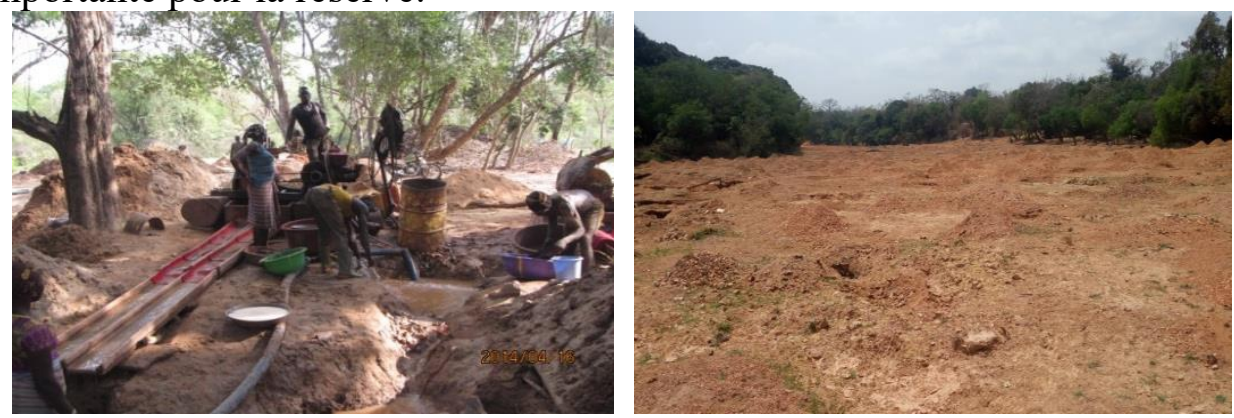

Figures 1 et 2: Sites d'orpaillage dans la réserve (clichés Dien, 2017)

\section{L'élevage}

L'élevage transhumant qui est le déplacement alternatif et saisonnier des animaux est un système de production animale largement pratiqué par les éleveurs de bovins dans la zone d'implantation de la réserve. Dans une région agricole comme celle du Haut Bandama où les besoins alimentaires d'entretien du cheptel ne sont pas couverts toute l'année selon les éleveurs, ces derniers, toujours à la recherche des pâturages et des points d'eau, ont recours à la réserve. Il s'agit de migrations de troupeaux de bœufs vers toutes les zones considérées comme zones de pâturage ou de parcage. Le fleuve Bandama à l'intérieur de la réserve sert de lieu d'abreuvement principal du bétail. A travers nos déplacements dans la réserve, nous avons pu constater la pression exercée par le bétail sur cette aire protégée, dans la mesure où ce sont des troupeaux pouvant dépasser une centaine de têtes qu'il nous a été donné de voir (figure 3). Cette présence des bœufs dans la réserve représente, selon les agents de l'OIPR, la deuxième menace sérieuse après l'orpaillage. Elle implique des acteurs nationaux et non nationaux, mais la gestion des troupeaux incombe généralement à des bouviers (bergers peulhs) qui sont considérés comme des professionnels dans le domaine. 

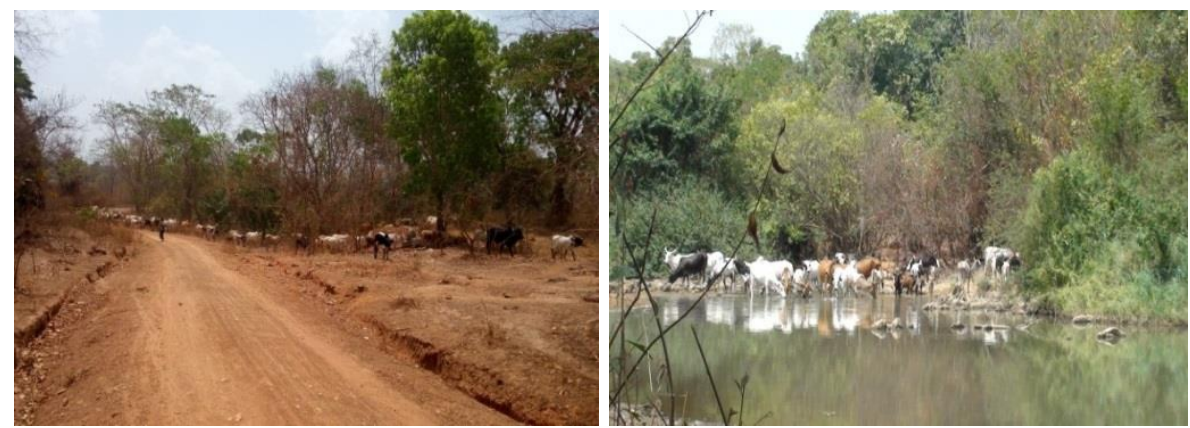

Figures 3 et 4 : Troupeaux dans la réserve (clichés Dien, 2017)

\section{L'agriculture}

La quasi-totalité des zones visitées est occupée à des degrés divers par l'activité agricole. Cette forme d'occupation est particulièrement liée à la culture de l'anacarde et implique des migrants allochtones et des allogènes. De gros campements comme Gbambalkaha, Siékaha, Siluélougokaha ou Kolokaha ont ainsi été érigés dans la réserve par des clandestins dont la plupart ont bénéficié de complicités locales, notamment celles des propriétaires terriens ou des chefs de village. Bien que la plupart de ces campements aient été détruits par l'OIPR, les plantations, elles, continuent d'être exploitées par leurs propriétaires. Aux dires des responsables de l'OIPR et des populations rencontrées, une mesure intervenue à la suite des négociations entre les deux parties en 2014 autorise l'accès des populations à ces plantations jusqu'en 2018, uniquement pour la récolte des noix d'anacarde. Cette mesure interdit l'entretien des champs et de nouveaux défrichements. Le constat est que la présence des populations dans la réserve, officiellement pour la récolte d'anacarde, favorise diverses formes d'agression de cet espace. Il s'agit principalement de l'utilisation d'herbicide dans les champs pour contourner l'interdiction de défrichement, de la pratique de la pêche dans le fleuve Bandama, et de la pratique de la chasse. Certains produits de pêche et de chasse sont même vendus par ces agriculteurs pour se faire des revenus.
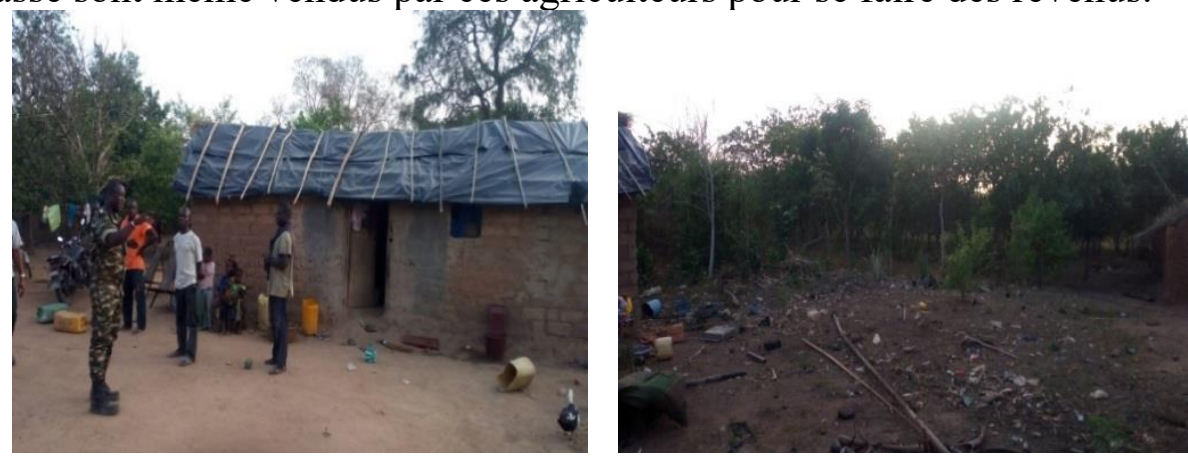

Figures 5 et 6 : Campements et plantations à l'intérieur de la réserve (clichés Dien, 2017) 


\section{Le braconnage}

Les populations locales assurent aussi une part importante de leur alimentation par le prélèvement d'animaux dans la réserve. La technique de chasse utilisée ici est la chasse au fusil qui se trouve encouragée par la grande disponibilité des armes à feu de fabrication traditionnelle. Aussi toutes les espèces animales de la réserve sont-elles visées, depuis les petits rongeurs jusqu'aux grands mammifères dont la présence, aux dires des populations, se fait rare. A partir des noms vernaculaires qui ont été cités, nous avons pu distinguer des primates (mamwi: chimpanzé, gbôgohon: cynocéphale, minanfi: singe au nez blanc, kôgninin: singe rouge, kôfili: singe blanc, kôwôlè : singe noir), des insectivores (kpoyoro : pangolin), des oiseaux (ntio : perdrix, triwale : calao) et des reptiles (wôtio : pithon). Mais les produits de chasse provenant de la réserve atteignent aussi souvent des niveaux importants de commercialisation et alimentent les marchés locaux (Katiola, Tortiya, Bouandougou, Marandalha, etc.) comme les marchés de plus grande envergure (Bouaké, Yamoussoukro et Abidjan). A ce sujet, les propos d'un notable de Namplékaha sont évocateurs: «quand vous mangez ça à Abidjan là-bas, vous pensez que ça quitte où ? Vous mangez ça bien à Abidjan et c'est nous les fournisseurs qui avons des problèmes.».

Il convient ici de souligner la collaboration entre les braconniers et les éleveurs Peulhs dans ce type de chasse à caractère commercial. Les derniers permettent aux premiers d'accéder aux sites giboyeux en leur fournissant des informations sur les animaux rencontrés et les lieux de rencontre; les premiers exploitent ces informations et, lorsque la chasse a été fructueuse, ils posent des actes de reconnaissance envers les derniers à travers le partage des revenus tirés de la vente du gibier.

Outre la commercialisation de gibier, le braconnage dans la réserve est encouragé par l'existence d'un marché local de produits dérivés d'animaux. Il s'agit, entre autres, des peaux et des cornes. La vente de ces produits procure des revenus importants aux chasseurs et aux revendeurs. Au marché de Katiola par exemple, il nous a été donné de constater que les peaux d'animaux en vente sont divisés en plusieurs morceaux de forme carré équivalant chacun à la somme de 1.000 FCFA $(1,52 €)$. 


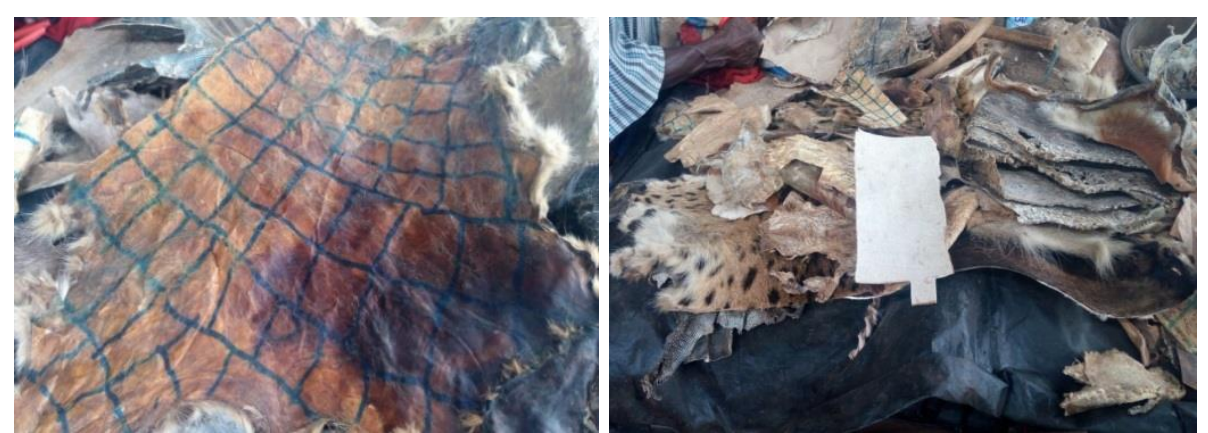

Figure 7 et 8 : Produits dérivés d'animaux vendus autour de la réserve (clichés Dien, 2017)

\section{La pêche}

La pêche est aussi pratiquée de façon artisanale sur le fleuve Bandama à l'intérieur de la réserve. On distingue ici la pêche professionnelle dont les produits sont destinés à la commercialisation et la pêche occasionnelle qui sert à l'autoconsommation ou à la commercialisation pour avoir des revenus d'appoint. Les acteurs de la pêche professionnelle sont les Bozos maliens installés dans les localités de Fronan et Niakaramandougou tandis que la pêche occasionnelle est le fait des populations des villages riverains. Toutes les techniques de pêche sont utilisées par ces personnes pour avoir le maximum de poissons (filets de petites mailles, utilisation de produits toxiques, etc.). Le caractère illégal de leurs activités se reflète dans la difficulté à réunir des données sur le volume des ressources halieutiques qu'ils prélèvent dans la réserve.

\section{La collecte de produits forestiers non ligneux}

Les activités de collecte des produits forestiers non ligneux dans la réserve du Haut Bandama concernent les produits qui ont une valeur nutritionnelle ou commerciale. Il s'agit des produits végétaux tels que le néré, le baobab, le karité et le tomi et des produits animaux comme l'escargot. Ces produits sont beaucoup convoités par les femmes qui en tirent des revenus substantiels. Selon les informations que nous avons recueillies, des acheteurs ambulants en provenance d'Abidjan ou de Bouaké sillonnent chaque année les villages riverains de la réserve pendant la saison du néré, du baobab, du karité ou de l'escargot. Les prix pratiqués aux populations sont les suivants :

- baobab (boite de $1,5 \mathrm{~kg}): 600(0,91 €)$ à $800 \operatorname{FCFA}(1,21 €)$

- néré (boite de 1,5 kg) : $750(1,14 €)$ à 1200 FCFA $(1,82 €)$

- $\quad$ karité (cuvette de $10 \mathrm{~kg}): 1500(2,28 €)$ à 3000 FCFA $(4,57 €)$

- $\quad$ escargot (sac de $50 \mathrm{~kg}): 6000(9,14 €)$ à 8000 FCFA $(12,19 €)$

La collecte de produits forestiers non ligneux dans la réserve du Haut Bandama porte également sur les plantes médicinales. 


\section{Les différents acteurs et leurs rôles}

Les différentes formes d'occupation de la réserve exposées plus haut laissent apparaître deux grandes catégories d'acteurs. Il s'agit des pourvoyeurs, d'une part, et des producteurs, d'autre part. Les pourvoyeurs sont les acteurs qui sont soit à l'origine des activités illicites dans la réserve soit organiseurs de ces activités soit facilitateurs. Ces personnes ne participent pas matériellement à la modification de la réserve mais, ils en constituent le ferment. Parmi elles, on note les commerçants d'or et de bœufs installés dans les zones urbaines nationales et dans les pays frontaliers, les personnes dites de pouvoir ou d'autorité (agents et/ou responsables des forces de défense sociale dont la réserve a été sous le contrôle avant la prise en main par l'OIPR, agents de l'OIPR), les chefs de village et les propriétaires terriens. Les commerçants (d'or et de bœufs) et les agents et/ou responsables des forces de défense sociale dont la réserve a été sous le contrôle constituent les véritables "actionnaires" des activités d'orpaillage et d'élevage dans la réserve. Ils assurent les conditions de ces activités, notamment le paiement des droits d'accès à la terre aux propriétaires terriens, la fourniture de matériel et de personnel et la prise en charge financière et alimentaire du personnel. Des "chefs de chantier" d'orpaillage et des éleveurs peuhls sont présents sur le terrain pour le compte de ces acteurs.

Les agents de l'OIPR constituent un maillon non négligeable des activités illicites dans la réserve. D'après nos informations, il semble, que c'est souvent avec la caution tacite et la bienveillance de ceux-ci que la réserve continue d'être occupée par les populations. Par exemple, pour un éleveur appréhendé avec un troupeau de bœuf dans la réserve, l'indulgence des agents de l'OIPR se négocie à hauteur de $100.000(152,44 €)$ à $150.000 \mathrm{FCFA}(228,67 €)$. Les relations de familiarité de ces agents avec les milieux de l'orpaillage, l'élevage et l'agriculture semblent évidentes quand on considère l'importance et la récidive de ces activités dans la réserve.

Les chefs de village et les propriétaires terriens sont des acteurs qui perçoivent des droits d'accès sur la terre et qui donnent leur accord pour l'installation des orpailleurs, des éleveurs et des agriculteurs dans la réserve. Du fait de la concentration du foncier entre ces quelques individus par village, les revenus monétaires provenant de la collecte des droits d'accès peuvent être considérables par an et par propriétaire.

Les producteurs, eux, sont les acteurs de terrain qui mènent des activités dans la réserve pour le compte des pourvoyeurs ou pour leur propre compte. Ils comprennent les travailleurs des "chantiers" d'orpaillage, les pêcheurs, les chasseurs, les éleveurs ou bergers peuhls, les agriculteurs migrants, les agriculteurs autochtones et les collecteurs de Produits Forestiers Non Ligneux. Sur les surfaces de terres qui ont été cédées aux paysans migrants dans la réserve, ceux-ci mettent en place un système d'exploitation rapide en utilisant 
des intrants. En outre, ils y construisent des campements de proximité. Tout comme les orpailleurs et les éleveurs, ils cherchent des alliances avec les autorités locales et l'administration pour conforter leur présence.

En raison du caractère clandestin et de la portée générale de leurs activités, tous ces acteurs peuvent faire l'objet d'une représentation cartographique sur l'ensemble de la réserve. Le tableau ci-dessous donne la répartition des acteurs principaux par zones d'activités.

Tableau I: Cartographie des acteurs de l'occupation de la réserve

\begin{tabular}{|c|c|c|}
\hline \multicolumn{2}{|c|}{ Zones d'activités } & Acteurs \\
\hline Sous-préfectures & $\begin{array}{c}\text { Situation / } \\
\text { RFFHB }\end{array}$ & $\begin{array}{c}\text { Agriculteurs, orpailleurs, pêcheurs, collecteurs de } \\
\text { produits forestiers non ligneux }\end{array}$ \\
\hline Fronan & Sud-Est & Agriculteurs, éleveurs, pêcheurs \\
\hline Niakaramandougou & Nord-Est & Agriculteurs, éleveurs \\
\hline Tortiya & Nord & Agriculteurs, éleveurs \\
\hline Boron & Nord-Ouest & Orpailleurs \\
\hline Marandallah & Ouest & Orpailleurs, agriculteurs \\
\hline Bouandougou & Sud-Ouest & \\
\hline
\end{tabular}

Sources: Enquêtes, 2017

La représentation spatiale des activités anthropiques dans la réserve est associée aux différents acteurs qui les génèrent. Les agriculteurs sont présents sur l'ensemble de la réserve, à l'exception de la zone ouest où cette présence est faible. Les orpailleurs ont pour lieux de prédilection les zones ouest et sudouest ; les éleveurs se rencontrent plus dans les zones nord, nord-est et nordouest; et les pêcheurs se localisent au sud-est et au nord-est. Quant aux collecteurs de produits forestiers non ligneux, ils se rencontrent particulièrement dans la zone sud-est.

\section{Les modes d'accès à la réserve}

Selon nos informations, la réserve ne faisant l'objet d'aucune véritable gestion administrative, son accès s'est fait suivant l'ordre et les circonstances d'arrivée des personnes. Pendant longtemps, les modes d'accès les plus courants ont été la cession gratuite et la libre intrusion pour les autochtones. Mais à la faveur de la crise sociopolitique en Côte d'ivoire, les infiltrations dans la réserve ont pris des formes spécifiques, en évoluant sensiblement vers des transactions onéreuses ou des intrusions forcées. Le Sous-préfet de Fronan exprime cette situation de la façon suivante : "En son temps, c'est le Général Thomas d'Aquin ${ }^{2}$ qui, aux dires des populations, les a orientées vers la zone, en leur disant d'y mettre uniquement les cultures vivrières. D'autres personnes se sont installées d'elles-mêmes comme il n'y avait pas de contrôle. Avec la guerre, ne sachant certainement pas le statut juridique de cette

\footnotetext{
${ }^{2}$ Ressortissant de Katiola et grande figure de l'armée ivoirienne, décédé en 1990
} 
réserve, les populations, surtout les jeunes, ont commencé à y créer des plantations. Il y aussi des étrangers qui ont été installés par des riverains moyennant des sommes d'argent. D'autres personnes se sont infiltrées de leur propre chef $\gg$.

Ainsi l'occupation humaine de la réserve du Haut Bandama se caractérise par une superposition de modes d'accès déterminés dans le temps.

\section{Les facteurs liés à l'occupation de la réserve}

D'après notre enquête, quatre principaux facteurs sont liés à l'occupation humaine de la réserve de Faune et de flore du Haut Bandama. Parmi ces facteurs figurent le dynamisme économique de la zone périphérique, la recomposition de cette zone face à la pression démographique et aux enjeux fonciers, la non intégration du statut juridique de la réserve et l'invisibilité des acteurs clés.

\section{Une réserve située dans une zone périphérique économiquement dynamique}

La réserve du Haut Bandama se trouve sur plusieurs terroirs qui regroupent les autochtones Sénoufo, Tagbana et Malinké (les bobos). Les Sénoufo et les Tagbana, majoritaires, se rencontrent dans les sous-préfectures de Niakaramandougou, Fronan, Tortiya et Boron tandis que les Malinké se trouvent dans les sous-préfectures de Marandallah et Bouandougou. Tous ces différents groupes ethniques se réclament propriétaires terriens.

L'économie locale, initialement basée sur l'agriculture, a connu une diversification, notamment avec l'intensification des activités minières et d'élevage de bovins à la faveur de la crise sociopolitique en Côte d'Ivoire. L'expansion de ces activités constitue le motif d'infiltration de la réserve. A l'origine, ce sont les populations résidentes qui y ont développé des pratiques agricoles extensives. Ensuite, elles y ont accueilli et installé des migrants à la recherche de terres cultivables, de l'or et d'espaces pastoraux. En termes de cultures de rente, les spéculations dominantes sont l'anacarde et le coton. Le coton est d'introduction ancienne dans la région. Il bénéficie de l'appui des structures comme CIDT, COIC, SECO et Ivoire coton qui distribuent les semences aux paysans à des prix subventionnés. Mais, après avoir connu une période faste, cette culture suscite actuellement très peu d'intérêt chez les populations à cause du coût de la production et du faible prix d'achat (265 FCFA $(0,40 €)$ le $\mathrm{kg}$ de coton de premier choix). A l'inverse, l'anacarde, avec une rente de 750 FCFA $(1,14 €)$ le $\mathrm{kg}$, est de plus en plus cultivé et se présente comme l'alternative la plus sûre pour les producteurs. Les principales cultures vivrières pratiquées par la population sont: le maïs, le riz, l'arachide, et l'igname. Ces cultures occupent également une place très importante en termes de revenus chez les populations de la région. Le riz et le maïs sont la nourriture 
de base. Les différents produits agricoles sont commercialisés sur place ou pendant les marchés ruraux hebdomadaires autour de la réserve.

Les activités d'élevage qui sont pratiquées par les populations s'articulent autour de plusieurs types de cheptels : bovins, ovins, caprins, porcins et volaille. L'élevage de bovins est principalement lié à la présence d'éleveurscommerçants, c'est-à-dire, des personnes ayant l'élevage comme activité principale et dont le cheptel est destiné à la vente. Cette catégorie est dominée par les allogènes (les Peuhls notamment). A côté de ces personnes, on trouve une autre catégorie qui est constituée d'autochtones Sénoufo et Tagbana, et qui pratique l'association agriculture-élevage. L'impossibilité de vivre uniquement de l'agriculture et la nécessité de disposer des bœufs pour la culture attelée conduisent à l'apparition de ces nouveaux acteurs. Comparés à l'élevage de bovins, les autres types d'élevage sont marginaux et permettent aux populations de faire face aux obligations sociales et religieuses (funérailles, dots, mariage, fête de la Tabaski, rituels, etc.) tout en étant une source de protéines.

Les activités d'orpaillage sont liées à l'installation d'une forte colonie d'allogènes venus de la sous-région ouest africaine. Elles créent un vaste mouvement de va-et-vient de chercheurs d'or dans la réserve et dans les localités environnantes. Mais, aujourd'hui, à cause de la baisse des prix des produits de rente et le vieillissement des plantations, les populations autochtones s'intéressent de plus en plus à cette activité.

\section{Une zone périphérique recomposée face à la pression démographique et aux enjeux fonciers}

En matière d'exploitation et d'utilisation des ressources naturelles dans la zone de la réserve de Faune et de Flore du Haut Bandama et sa périphérie, les éléments qui appartiennent à toute la communauté sont l'herbe, l'eau et les produits des arbres non plantées (le karité ou le baobab par exemple). La terre, au contraire, a toujours un propriétaire et les rapports des individus à cette ressource sont régis par la coutume. Celle-ci reconnaît aux Sénoufo, Tagbana et Malinké des droits de propriété et d'exercice, du fait de leur qualité de premiers installés dans la zone.

Par le passé, l'accès à la terre était consécutif à l'héritage ou à un don symbolique au chef de terre (la cola ou un poulet généralement) pour acquérir un droit de cultiver ou de s'installer sur une parcelle. Mais aujourd'hui ces mécanismes propres à la société traditionnelle se sont altérés en raison de l'augmentation de la population et de la forte pression foncière qui concerne, principalement, la culture de l'anacarde, l'élevage et l'orpaillage. Aussi, les propriétaires terriens ont-ils institué une autre forme d'accès à la terre qui est relative au paiement d'une redevance annuelle variant de $10.000(15,24 €)$ à 20.000 FCFA $(30,48 €)$ par exploitant et, dans certains cas, par hectare. Cette 
redevance est parfois accompagnée d'une partie de la production agricole annuelle. Par ailleurs, le développement de la culture de l'anacarde, et dans une moindre mesure, celle de la mangue, a favorisé une distinction en matière de cession du foncier, en faisant la part entre le droit de pratiquer des cultures annuelles et celui de pratiquer des cultures pérennes.

La pression foncière autour de la réserve engendre des conflits entre des villages à propos des limites des territoires villageois, entre les propriétaires terriens et les utilisateurs dont certains ne respectent pas les règles d'accès, et entre agriculteurs et éleveurs au sujet des dégâts de cultures et de récoltes. Pour gérer ces conflits, les protagonistes ont recours à divers mécanismes comme le règlement par consensus entre les parties ou le règlement au niveau des autorités coutumières (chef de terre, chef de clan, chef de canton, Imam, etc.). Dans les villages où il existe une Commission Villageoise de Règlement à l'Amiable, les dissensions mineures sont résolues par cette instance. L'administration (le Sous-préfet et, le cas échéant, les services de l'agriculture) est sollicitée en cas de conflits ou de dégâts graves et, surtout, lorsqu'il s'avère impossible pour les autorités coutumières ou la Commission Villageoise de Règlement à l'Amiable de concilier les protagonistes.

\section{Une réserve au statut juridique non intégré par les populations}

La réserve de faune et de flore du Haut Bandama est une aire protégée dont la création remonte à un décret pris en 1973 (décret $\mathrm{n}^{\circ} 73-133$ du 21 mars 1973), c'est-à-dire 29 ans avant l'adoption de la loi n²002-102 du 11 février 2002, relative à la création, à la gestion et au financement des parcs nationaux et des réserves naturelles. En février 2014, lorsque 1'administration de l'OIPR, après la création d'une base à Katiola, amorce effectivement la gestion de cette réserve, cela faisait pratiquement 40 ans que cette dernière est restée en retrait de la politique nationale de conservation des aires protégées. La longue absence de l'administration forestière, selon Akindes et al. (2016) a laissé le temps aux populations de la périphérie de penser, pour certaines, que le statut de réserve, donc d'aire interdite de toute présence et d'activités humaines, n'était qu'une vue de l'esprit, et pour d'autres, qu'il n'était plus d'actualité. Ainsi à divers titres, les différentes ressources de cet espace ont fait l'objet d'exploitation par plusieurs catégories d'acteurs qui, à la longue, ne se percevaient plus vraiment comme des exploitants illégaux.

\section{Des acteurs clés invisibles}

Bien qu'anarchiques à première vue, les activités humaines qui s'exercent dans la réserve sont très structurées et hiérarchisées surtout en ce qui concerne l'orpaillage et l'élevage. Derrière ces activités aux enjeux importants, se cachent le plus souvent des dirigeants qui occupent des positions stratégiques et qui se conduisent en véritables défenseurs des acteurs sur le terrain. Selon 
nos informations, les pratiques de ces personnes reposent sur un réseau de communication bien organisé et des accointances avec les milieux administratif, politique et judiciaire. Ainsi, dès qu'une patrouille est programmée à l'intérieur de la réserve par l'OIPR depuis la direction de zone à Yamoussoukro, l'information est immédiatement accessible aux acteurs de terrain qui prennent rapidement des dispositions conséquentes. Lorsque ces derniers sont appréhendés, ils ne subissent généralement pas les effets juridiques de leurs actes vu que les dirigeants font tout pour obtenir leur libération souvent en échange d'un montant d'argent. La persistance de cette réalité liée au contrôle des activités humaines dans la réserve par des acteurs clés invisibles est déterminante dans la portée actuelle de la dégradation de la biodiversité de cette aire protégée.

\section{DISCUSSION}

Les résultats qui précèdent mettent en évidence des aspects induits par l'homme, et qui relèvent d'une demande socioéconomique des ressources de la réserve de Faune et de flore du haut Bandama. Les formes d'activités anthropiques recensées dans notre étude sont l'orpaillage, l'élevage, l'agriculture, le braconnage, la pêche et la collecte de produits forestiers non ligneux. Sur la base de ces données, nous retiendrons la typologie de Kangbéni et al. (2012) : trois formes d'activités relèvent des activités extractives qui s'exercent soit sur la faune (braconnage et pêche) soit sur la flore (collecte de produits forestiers non ligneux), tandis que trois autres concernent l'occupation de l'espace protégé et sa transformation à des fins anthropiques (orpaillage, élevage, agriculture). Ceci confirme les résultats de l'étude d'évaluation de l'efficacité de la gestion des aires protégées en Côte d'Ivoire (2008) qui identifie ces types d'activités comme étant les principales pressions anthropiques qui s'exercent sur les Parcs et réserves de Côte d'Ivoire. Mais la comparaison de nos résultats et ceux obtenus dans le cadre de cette étude permet aussi de conclure à la stabilité des activités constitutives de l'occupation humaine de la réserve. L'identification des acteurs impliqués dans le processus d'exploitation des ressources de cette aire protégée et la caractérisation des interactions qui entourent leurs activités met en évidence l'existence de pourvoyeurs, c'est-à-dire les acteurs clés qui contrôlent ce processus, et les producteurs, c'est-à-dire ceux qui mènent les activités sur le terrain. Les relations sociales d'interdépendance au sein de ces deux catégories d'acteurs se rapprochent de celles des réseaux criminels décrites par LavaudLegendre et al. (2017) dans le cadre de leur étude sur la traite des êtres humains. Ce qui laisse supposer que la criminalité environnementale liée à l'occupation humaine de la réserve de Faune et de Flore du Haut Bandama, au-delà des caractéristiques qu'on retrouve dans les autres aires protégées de 
Côte d'Ivoire, recèle des particularités qui relèvent de la présence de réseaux criminels.

L'analyse des principaux facteurs à l'origine de ce phénomène semble révéler deux situations. D'une part, on pourrait penser que le dynamisme économique de la zone périphérique et sa recomposition face à la pression démographique implique une dépendance des populations vis-à-vis des ressources de la réserve. D'autre part, on peut dire que la non intégration du statut juridique de la réserve et l'invisibilité des acteurs clés dissimule des problèmes pratiques de gestion de la réserve. Ces idées se rapprochent des travaux de Koffi (2016) qui accordent une large place à la dépendance des populations locales en ressources forestières dans l'explication de l'exploitation des aires protégées par celles-ci ; laquelle exploitation induit de réels problèmes de gestion de ces sites.

\section{CONCLUSION ET PERSPECTIVES}

L'occupation humaine de la réserve de Faune et de Flore du Haut Bandama constitue une contrainte majeure au regard des objectifs de conservation de cette aire protégée. Cette occupation continue de se manifester sous plusieurs formes, dont l'orpaillage, l'élevage, l'agriculture, le braconnage, la pêche et la collecte de produits forestiers non ligneux. La nature des rapports des acteurs et leurs rôles complémentaires donnent à ces activités illégales les caractéristiques d'un secteur organisé. Parmi les facteurs qui influencent l'accentuation de ces pressions sur la réserve, on note le dynamisme économique de la zone périphérique, la recomposition de cette zone face à la pression démographique et aux enjeux fonciers, la non intégration du statut juridique de la réserve et l'invisibilité des acteurs clés. Pour escompter pouvoir agir sur ces facteurs, les actions qui paraissent être nécessaires à réaliser sont de trois ordres : la mise en place d'une politique de gestion sensible aux questions sociales et économiques, la participation des riverains dans la prise de décision, et la coopération avec les partenaires et les structures régionaux ou nationaux.

\section{La mise en auvre d'une politique adéquate}

La réserve, au vu de ce qui précède, constitue actuellement un enjeu tant pour les gestionnaires que pour les populations riveraines utilisatrices des ressources naturelles qu'elle renferme. Même si les activités d'information, d'éducation, de coopération ou de répression mises en œuvre par l'OIPR dans le cadre de la reprise en main de cet espace ont enregistré des résultats positifs, force est de constater qu'elles n'ont véritablement pas encore permis de le soustraire aux impacts de l'occupation illégale par ces populations. Au stade présent, et face à l'importance et au caractère ancien de l'anthropisation de la réserve, il ne serait pas exagéré de dire que les moyens de subsistance des 
populations locales dépendent largement de cette aire protégée. Dans ce contexte, la principale contrainte qu'induit la perspective de sa conservation est, comme le soulignent Akindes et al. (2016) celle de l'insécurité des revenus des producteurs agricoles et des opérateurs économiques exerçant dans des filières de spéculations qui s'y trouvent. Car pour ces personnes qui, durant des années, ont vécu de l'exploitation des ressources de la réserve, leur départ des sites occupés suppose une perte de sources de revenus pour certains ou une baisse de revenus pour d'autres. Il apparaît donc essentiel que les autorités en charge de cette aire protégée s'engagent dans la voie de la recherche de solutions allant plus loin que les mesures légales actuellement en vigueur. Par exemple, il serait pertinent de distinguer les activités qui sont liées à la pauvreté dans la zone riveraine des activités qui relèvent de la convoitise et de l'appât du gain. Ainsi, sur cette base, l'OIPR pourrait intégrer les dimensions sociale et économique dans sa stratégie de dissuasion en mettant en place un cadre de politique d'accompagnement des personnes affectées. Même s'il n'est pas de son ressort de s'occuper de relocalisation, il serait souhaitable que cette structure apporte des appuis au développement socioéconomique de ces populations à travers des programmes d'investissements orientés vers des activités compatibles avec l'utilisation durable de la biodiversité à l'intérieur de la réserve.

\section{La participation des riverains}

L'implication des populations riveraines ou infiltrées revêt une importance capitale dans la conservation de la réserve. Dans le cadre de sa stratégie de gestion, il nous paraît nécessaire que l'OIPR identifie les acteurs clés pouvant jouer un rôle fondamental dans la prévention et l'atténuation des menaces visà-vis de la réserve, leur apporter un appui institutionnel, et renforcer leurs capacités en matière de protection de l'environnement. Ces acteurs pourront être des personnes généralement très écoutées par les populations à savoir les préfets et sous-préfets des zones concernées, les ONG de l'environnement, les ONG de droits de l'homme, les chefs de canton, les chefs de village, les représentants d'associations de jeunesse ou de femme, les propriétaires terriens, et les représentants des populations illégalement installées dans la réserve. Ensuite, l'OIPR veillera à mettre en place avec ces personnes un cadre de concertation et de prise de décision participative sur les opérations de déplacement involontaire et d'accompagnement des populations illégalement installées dans la réserve. Ce cadre de concertation pourrait comprendre également les représentants des ministères de l'environnement et de l'intérieur. 


\section{La coopération régionale et nationale}

L'identification des divers acteurs et des utilisations illicites de la réserve a permis de mettre en évidence la portée de ces activités qui va parfois au-delà des capacités de l'OIPR. Une réponse à cette situation est que l'OIPR s'engage dans la voie de la coopération régionale et nationale. Des mécanismes de collaboration en matière d'enquête et de recherche des auteurs d'infractions sur la réserve pourraient ainsi être mises en place à travers des protocoles d'accords et des structures spécialisées. A ce sujet, le renforcement de la coopération avec les partenaires et les autres structures de défense sociale semble être une action indispensable.

\section{References:}

1. AGOUALE, Y. J. (2013). Contribution de la télédétection et du SIG à la gestion intégrée de la réserve du Haut Bandama. Mémoire de Master, UFR : Communication, Milieu et Société, Université Alassane Ouattara.

2. AKINDES, F., SEDIA, G., FOFANA, M. (2016). Aspects socioéconomiques, Parc national des Iles Ehotilé, Réserves naturelles d'Abokouamékro, de Dahliafleur, du Haut Bandama, de Lamto et du Mont Nimba, phase finale du bilan de l'état de la biodiversité des aires protégées de Côte d'Ivoire, rapport provisoire

3.BERD-E\&D Consult (2014). Etude d'impact social du projet de conservation des ressources naturelles de Côte d'Ivoire, rapport final

4. GIROIR, G. (2007). Les impacts anthropiques dans les parcs nationaux chinois : approche géographique, Géocarrefour, vol. 82/4, 187-197

5. GOH, D. (2016). L'exploitation artisanale de l'or en Côte d'Ivoire: la persistance d'une activité illégale. European Scientific Journal January vol.12.

6. KABORE, A. (2010). Brousse des uns, aire protégée des autres Histoire du peuplement, perceptions de la nature et politique des aires protégées dans le Gourma burkinabè: 1'exemple de la Réserve partielle de faune de Pama. Thèse présentée à l'Institut de Hautes Etudes Internationales et du Développement pour l'obtention du grade de Docteur en études du développement, Burkina Faso.

7. KANGBENI, D., KPERKOUMA, W., KOMLAN, B., MARRA, D., YAO A W. ET KOFFI, A. (2012). « Analyse spatiale des différentes formes de pressions anthropiques dans la réserve de faune de l'OtiMandouri (Togo) », VertigO - la revue électronique en sciences de l'environnement [En ligne], Hors-série 14|, mis en ligne le 15 septembre 2012, consulté le 28 juillet 2018. 
8.URL : http://journals.openedition.org/vertigo/12423 ; DOI : 10.4000/vertigo. 12423

9. KOFFI, K.Y. (2016). Le droit des aires protégées en Côte d'ivoire. Thèse de doctorat, Université de Maastricht.

10. KOUAME, N. F., KOUASSI, K. E., KOUADIO, K., AHIMIN, A. O. (non daté). Zones d'importance écologique particulière et valorisation de la biodiversité.

11. LAVAUD-LEGENDRE, B., PLESSARD, C., LAUMOND, A., MELANÇON, G., PINAUD, B. (2017). Analyse de réseaux criminels de traite des êtres humains: méthodologie, modélisation et visualisation. Graphes \& systèmes sociaux, volume 2 .

12. MENGUE-MEDOU, C. (2002). Les aires protégées en Afrique : perspectives pour leur conservation, VertigO - la revue électronique en sciences de l'environnement [En ligne], Volume 3 Numéro 1 | avril 2002, mis en ligne le 01 avril 2002, consulté le 16 mars 2018.

13. MANIRABONA, A. \& KOUTOUKI, K. (2016). Introduction : La criminalité environnementale. Criminologie, 49(2), 5-14. doi:10.7202/1038414ar

14. Office des Nations Unies contre la drogue et le crime (2012). Compilation d'outils pour l'analyse de la criminalité liée aux espèces sauvages et aux forêts.

15. Office Ivoirien des Parcs et Réserves (2016). Manuel d'exécution des mesures riveraines, version définitive.

16. Stratégie de Relance du développement et de Réduction de la Pauvreté (DSRP), (2009). République de Côte d'Ivoire.

17. TETRA TECH (2017). Diagnostic Ecologique et Socio-économique de la Réserve de Faune et de Flore du Haut Bandama.

18. UICN/BRAO (2008). Evaluation de l'efficacité de la gestion des aires protégées : parcs et réserves de Côte d'Ivoire.

19. VEÏ, K.N. (2015). Dynamique spatio-temporelle de la réserve du Haut Bandama en Côte d'Ivoire. In Germivoire $n^{\circ}$ 2/2015, URL: http://germivoire.net/?ivoire=detailart\&idart $=415 \& \mathrm{rub}=152$. 